\title{
Factory-Calibrated Continuous Glucose Sensors: The Science Behind the Technology
}

\author{
Udo Hoss, PhD and Erwin Satrya Budiman, PhD
}

\begin{abstract}
The use of commercially available continuous glucose monitors for diabetes management requires sensor calibrations, which until recently are exclusively performed by the patient. A new development is the implementation of factory calibration for subcutaneous glucose sensors, which eliminates the need for user calibrations and the associated blood glucose tests. Factory calibration means that the calibration process is part of the sensor manufacturing process and performed under controlled laboratory conditions. The ability to move from a user calibration to factory calibration is based on several technical requirements related to sensor stability and the robustness of the sensor manufacturing process. The main advantages of factory calibration over the conventional user calibration are: (a) more convenience for the user, since no more fingersticks are required for calibration and (b) elimination of use errors related to the execution of the calibration process, which can lead to sensor inaccuracies. The FreeStyle Libre ${ }^{\mathrm{TM}}$ and FreeStyle Libre Pro ${ }^{\mathrm{TM}}$ flash continuous glucose monitoring systems are the first commercially available sensor systems using factory-calibrated sensors. For these sensor systems, no user calibrations are required throughout the sensor wear duration.
\end{abstract}

Keywords: Continuous Glucose Monitoring, Glucose sensor, Calibration, Factory calibration, Subcutaneous.

\section{Introduction}

$\mathbf{S}^{\mathrm{s}}$ NCE THE INTRODUCTION of commercially available continuous glucose monitoring systems in 2000, significant progress in terms of system performance and convenience of use has been achieved, garnering positive expectation on the clinical utility and adoption of this technology. ${ }^{1,2}$ The first system introduced by MiniMed was a retrospective system, with data being available to the user or healthcare professional at the end of the sensor wear time. ${ }^{3}$ These early sensors could only be used for up to 3 days and needed a minimum of four calibrations per day. Over the following years the systems became easier to use, the accuracy of the systems improved, and the allowed wear duration was extended. However, until recently all systems still required daily blood glucose (BG) tests for recalibration to maintain accurate sensor glucose readings throughout sensor wear.

Most currently available continuous glucose monitoring systems employ enzymatic amperometric sensors measuring glucose in the interstitial subcutaneous tissue. The measure- ment signal is an electrical current. That current is proportional to the glucose concentration at the measurement site, with a small background current, which can be accounted for as a signal offset if necessary. To display glucose information to the user of the system, the sensor signal will have to be converted from an electrical current to a glucose value. This conversion is called calibration, and involves a BG test by the user. Assuming a linear sensor response to glucose and a negligible or known background signal, the sensor sensitivity to glucose can be calculated from one sensor current value and its corresponding time-matched BG reading. The sensor sensitivity represents the calibration factor, which can be used to convert the sensor electrical response into a glucose value moving forward from the calibration time point.

The user calibration process has several disadvantages. First, it is a burden to the user of the sensor system, since each calibration process requires a painful and time-consuming BG test. More importantly, the accuracy of the BG test directly determines the accuracy of the sensor system. Certain user mistakes like not washing hands before a BG test can

Abbott Diabetes Care, Alameda, California. 
lead to wrong glucose numbers. Some sensor systems require the user to enter the BG value manually for calibration, where transcription error and delayed BG entry can affect sensor accuracy. On the other side, assuming the BG test was performed correctly, if at the time of calibration the sensor signal has a temporarily falsely reduced or elevated value, for example, caused by interfering substances, the calculated sensor sensitivity will not be correct and the following sensor data will persistently be falsely reduced or elevated. An alternative to the user calibration process is sensor factory calibration, which has been implemented for the FreeStyle Libre ${ }^{\mathrm{TM}}$ and FreeStyle Libre Pro ${ }^{\mathrm{TM}}$ flash continuous glucose monitoring systems (hereafter referred to as FreeStyle Libre).

In this article, we will review data to be collected through scientific experiments to demonstrate the feasibility of factory calibration. These requirements apply to any subcutaneous glucose sensor system intended to be factory calibrated. Results obtained during the development of FreeStyle Libre are included to illustrate the type of experiments and analysis required. However, it is not the purpose of this article to provide comprehensive data demonstrating feasibility of factory calibration for the FreeStyle Libre sensor.

\section{What is Factory Calibration?}

Factory calibration of sensors removes the need for determining the sensor sensitivity from the user's responsibility and instead places it in the hands of the sensor manufacturer. The sensor sensitivity is determined during the sensor manufacturing process, and that information is included with every sensor in the form of a sensor code. That code can be preprogrammed into the sensor electronics such that no user interaction is required to enter the code, eliminating the risk of transcription error.

The factory calibration process includes the following steps:

- Manufacture sensor lots with low sensor to sensor variability.

- Sample a number of sensors from each sensor lot and test them in the laboratory (in vitro) for their response to glucose and determine their glucose sensitivity.

- Convert the lot glucose sensitivity into a sensor code.

- Program the sensor code into the sensor electronics memory.

- Demonstrate that the initially determined sensor sensitivity does not change over the sensor shelf life.

Since the variation between the sensors in one sensor lot is small, the laboratory tested sensors are representative of the remaining sensors in the sensor lot, which will be used by patients. The code information provides the necessary sensor sensitivity or calibration factor for every sensor in the sensor lot to convert the electrical sensor current into a glucose value. The determination of the code may include corrections for the difference between in vitro and in vivo sensor testing, which can be determined analytically or empirically through clinical trials, and which can be applied universally to all sensor lots.

This process determines how the sensor responds to glucose and will provide glucose data after sensor insertion without the necessity of a BG test by the user. It does, however, by itself not remove the requirement for recalibrations during sensor wear. To avoid recalibrations, it is necessary that the assigned sensor sensitivity remains valid throughout the sensor wear. This is a requirement related to both the sensor chemistry as well as the sensor biocompatibility.

The term factory calibration refers by itself only to the process of determining the initial sensor sensitivity during the manufacturing process. However, it is widely understood and expected that factory-calibrated sensors do not require any calibrations by the user, including no recalibrations during sensor wear.

To be able to provide factory-calibrated sensors to the user there is a set of requirements beyond the general requirements shared among glucose sensors, ${ }^{4}$ as outlined in Table 1.

The first three requirements are related to the design and manufacturing of the sensor and the chemistry involved, whereas the last requirement depends on the biology of the interstitial tissue.

With respect to consistency of the sensor manufacturing process, it is important to identify the sensor components which do affect its sensitivity. For an amperometric sensor, the sensing area located on the working electrode containing the enzyme and the membrane covering the enzyme and limiting the flux of glucose from the tissue to the enzyme are the critical components. Therefore, it is essential to develop processes to reproducibly deposit the enzyme on the working electrode and to create a uniform coating of the glucoselimiting membrane. Variations in sensing layer area and membrane thickness between sensors have to be kept small, which requires a high-precision manufacturing equipment given that the areas involved are in the range of less than $1 \mathrm{~mm}^{2}$ and the membrane thickness is typically less than $100 \mu \mathrm{m}$. Sensor design and architecture determine the options for manufacturing methods. Therefore, if factory calibration is the goal, it is crucial that these limitations are taken into consideration early in the development process, so that the sensor architecture will allow the use of robust manufacturing processes.

The sensor sensitivity is determined as part of the factory calibration process at the end of the sensor manufacturing process. This information is assigned to every individual sensor usually in the form of a code. However, sensors are not being used immediately after they are produced, and there will be a period of time between the production and the use date. During that time the sensor sensitivity cannot change. Otherwise, the initially assigned sensitivity is no longer valid and the sensor will provide false data once inserted and used by the patient.

Table 1. Requirements for Factory Calibration

\begin{tabular}{lcc}
\hline Requirement & \multicolumn{1}{c}{ Objective } & $\begin{array}{c}\text { Testing } \\
\text { environment }\end{array}$ \\
\hline $\begin{array}{c}\text { Consistent sensor } \\
\text { manufacturing }\end{array}$ & $\begin{array}{c}\text { Reduce sensitivity } \\
\text { variation between } \\
\text { sensors. } \\
\text { Shelf life stability } \\
\text { Maintain sensor } \\
\text { sensitivity over the } \\
\text { assigned shelf life. } \\
\text { Wear stability }\end{array}$ & In vitro \\
& $\begin{array}{c}\text { In vitro } \\
\text { sensitivity over the } \\
\text { wear duration. }\end{array}$ & $\begin{array}{c}\text { In vitro/ } \\
\text { in vivo }\end{array}$ \\
$\begin{array}{c}\text { Consistent } \\
\text { blood/tissue } \\
\text { relationship }\end{array}$ & $\begin{array}{c}\text { Demonstrate consistent } \\
\text { BG-to-ISF-glucose } \\
\text { gradient between } \\
\text { subjects. }\end{array}$ & In vivo \\
\hline
\end{tabular}

BG, blood glucose. 
Similar to the requirement that the sensor needs to be stable over its assigned shelf life, it also needs to be stable over its use period. ${ }^{5}$ If the sensitivity of the sensor to glucose does not change over the wear time, then recalibrations are not necessary. Alternatively, if the sensor response does change, recalibrations by the user can compensate for that sensor drift. Sensor drift is the reason why all currently available sensor systems, except FreeStyle Libre, require BG-based fingerstick calibrations by the user, typically twice a day.

The sensor stability over its use period is determined by two fundamentally different sensor properties. The first is the ability of the sensor to detect glucose with a stable sensor response. This property is governed by the underlying sensor chemistry and the enzyme involved, and can be demonstrated through in vitro tests. The second property is related to the biocompatibility of the sensor. The foreign body response to the sensor inserted into the subcutaneous tissue may lead to a change in sensor response. ${ }^{6}$ Therefore, to keep the signal stable over the wear period, the sensor design and the membrane chemistry either have to minimize the foreign body response or be able to prevent that response from interfering with the sensor signal. The sensor stability in vivo has to be determined through clinical trials.

The last requirement for the feasibility of factory calibration is the only requirement that is not related to the sensor itself. Since the currently available sensor systems are measuring glucose in the interstitial fluid, but are expected to predict the BG concentration, a consistent ratio between blood and tissue glucose is required. Many studies have been performed to estimate the absolute value of the interstitial glucose concentration and its relationship to BG. No clear consensus has been achieved to date, ${ }^{7}$ but most recent publications tend to estimate the tissue glucose to be around $90 \%$ of BG under steady-state conditions. ${ }^{8}$ However, most studies only report an average value and attribute any variations to the experimental conditions and errors. Therefore, data need to be generated with respect to the variation of the blood to tissue glucose ratio variation both within a subject at different body sites or between different subjects.

The accuracy of a factory-calibrated sensor system will depend on the variations of the parameters associated with the requirements in Table 1 . For example, minimizing the variation in sensitivity from sensor to sensor through manufacturing controls will minimize the sensor performance variance from individual to individual. The overall accuracy of the factory-calibrated sensor is achieved by applying appropriate specifications for the requirements in Table 1. Each specification will impact the accuracy independently, and therefore, it is up to the manufacturer to choose a set of specifications which will guarantee a desired accuracy level.

\section{Implementation of Factory Calibration for FreeStyle Libre}

The FreeStyle Libre and FreeStyle Libre Pro flash continuous glucose monitoring systems are the first commercially available factory-calibrated sensor systems. To our knowledge, no scientific studies have been published previously evaluating the feasibility of factory calibration besides the ones leading to FreeStyle Libre. ${ }^{9-13}$ All calibration-related studies and publications were focused on understanding and improving the standard BG-based fingerstick calibration ${ }^{14-30}$ or overcoming transient effects, such as lag and signal artifacts $^{31-35}$ that can impact calibration. This demonstrates the novelty of this alternative approach and also possibly the superiority of the chemistry used in FreeStyle Libre over other sensor systems.

The development of the FreeStyle Libre sensor was guided by the requirements as outlined in Table 1 . The chemistry as well as the architecture of the sensor was optimized to provide the necessary stability and robustness.

The FreeStyle Libre sensor is an enzymatic amperometric 3-electrode sensor system. The chemistry is based on the Wired Enzyme technology, which has been utilized in the FreeStyle Navigator continuous glucose monitoring system. This technique uses mediator molecules which are crosslinked together with the enzyme into a polymer matrix. Glucose molecules diffuse from the interstitial tissue through the outer membrane into the enzyme matrix and are oxidized by the enzyme glucose oxidase. The resulting electrons are transferred from the enzyme to mediator molecules (an osmium complex) and then shuttled to the working electrode using neighboring mediator molecules. The required electrical potential at the working electrode is only $40 \mathrm{mV}$ versus a $\mathrm{Ag} /$ $\mathrm{AgCl}$ reference electrode. A low electrical potential minimizes the oxidation of electroactive species at the working electrode and thereby minimizes susceptibility to interferents. ${ }^{36-38}$

The sensor design and the related manufacturing processes for the FreeStyle Libre sensor were chosen specifically to be able to manufacture identical sensors with respect to their response to glucose (Table 1). The most critical elements are the sensing layer containing the enzyme and the glucose limiting membrane. The manufacturing equipment applying these two components has been optimized for robustness and reproducibility. Additional inspection steps ensure that every single sensor meets the predetermined specification criteria. Sensor lot release testing provides the lot calibration code and also includes a quantitative measure of within-lot variability.

The factory calibration process is based on the assumption that the in vitro sensor sensitivity predicts the in vivo sensor response. Since the sensor measurement site is the interstitial fluid and the reported value is BG, it is required to establish the relationship between the glucose concentrations of these two compartments. This can be done analytically or empirically. The analytical path will take into account all factors which are different in vitro versus in vivo, and which do influence the sensor response, for example, absolute glucose concentration, temperature, oxygen, and interfering substances. Alternatively, the in vitro to in vivo relationship can be established empirically by performing clinical studies and comparing the in vivo response to the in vitro data.

For example, the in vitro sensitivity can be calculated by examining the signal response of a sample of sensors from a lot to a set of known glucose concentrations, and then calculating the in vitro glucose sensitivity for each sensor. The nominal in vitro sensitivity of that sensor lot is then determined by taking the mean of the per-sensor in vitro sensitivities. Similarly, the in vivo sensitivity can be calculated by examining all the paired sensor/reference BG values in each sensor from a clinical study, and calculating the in vivo sensitivity for each of the sensors. Finally, the pooled in vivo sensitivity of that sensor lot is calculated by taking the mean of the per-sensor in vivo sensitivities. 
FIG. 1. Correlation between mean in vitro sensitivity and mean in vivo sensitivity of sensors from sensor lots used in Study 1 and 2. Mean in vitro sensitivity (horizontal axis) is the lot average of the individual in vitro sensor responses (sensor signal in $\mathrm{nA}$ divided by glucose concentration in $\mathrm{mM}$ ). The in vitro sensor response was determined by testing sensors in glucose solution $(20 \mathrm{mM}$ phosphate buffered saline) with glucose concentrations ranging from 1 to $30 \mathrm{mM}$. The corresponding mean in vivo sensitivity (vertical axis) was obtained from clinical data. Individual in vivo sensor responses were calculated using capillary BG values and time paired sensor values. Correlation between in vitro and in vivo sensor sensitivity makes it possible to predict the in vivo sensor response from in vitro sensor testing (factory calibration). BG, blood glucose.

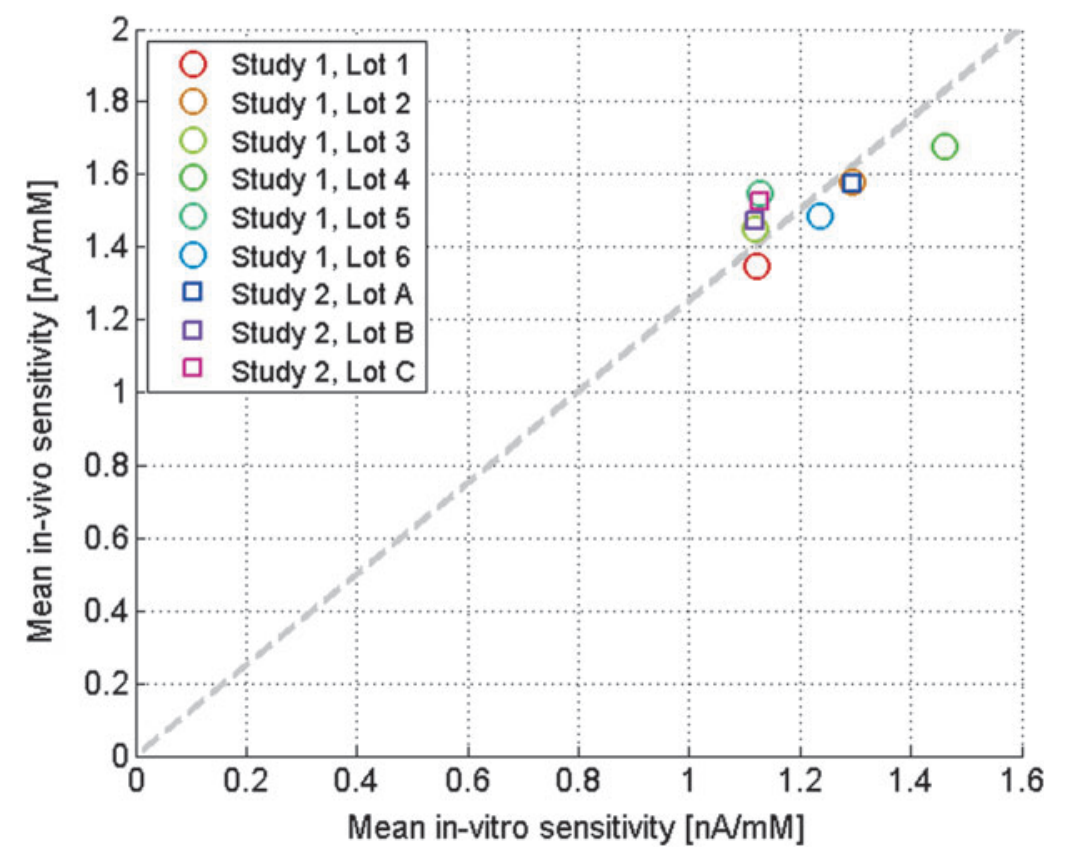

Figure 1 shows the average in vivo sensor response from multiple sensor lots compared with their in vitro sensitivity. Data shown are drawn from two separate clinical studies. One study was performed in 12 subjects with diabetes, each subject wearing three sensors from six different sensor lots simultaneously over a 5-day wear period (Fig. 1: Study 1, lot 1 through 6). The other study includes 72 subjects with diabetes, ${ }^{12}$ each subject wore two sensors simultaneously. A total of three sensor lots were evaluated in this study (Fig. 1: Study 2, lot A through C). Capillary BG values are used as the reference BG in this analysis. While the study population and timing of the studies may have an influence on the sensitivity values and the narrow sensitivity range of the sensor lots used in the studies is limiting the statistical significance of data, we can see a correlation between the in vitro and in vivo values and an overlap of data from the two separate clinical studies.

Measuring and monitoring shelf-life stability for sensors can be performed under standard temperature conditions or under accelerated conditions at elevated temperatures. If accelerated conditions are chosen, data need to be available to determine the required exposure duration at the selected elevated temperature. These data are usually based on an Arrhenius relationship, which needs to be established for the specific sensor system. Sensor shelf life is limited by the stability of the enzyme and it is essential that the enzyme immobilization conditions are selected carefully. For the FreeStyle Libre sensor, the enzyme is immobilized in a crosslinked polymer matrix, which provides an optimized environment for enzyme stability. ${ }^{39}$

Sensor stability for the FreeStyle Libre sensor over its 14day use period has been demonstrated earlier. ${ }^{10}$ The in vitro tests include an initial sensitivity test, where the sensor is exposed to glucose solutions with different glucose concentrations. From the sensor response, a sensitivity value can be calculated. After this initial test, the sensors are kept in a glucose solution for 14 days to measure that glucose level continuously. After the 14-day period, another sensitivity test equal to the test at the beginning is being performed, and the resulting sensitivity is compared with the sensitivity at the beginning of the 14-day test. The difference between the initial and the final test represents the drift the sensor is experiencing over a 14-day monitoring period.

In vivo testing of sensor stability is absolutely required in addition to in vitro testing since different processes may be limiting stability in the tissue. In vivo stability is the ultimate requirement for sensor stability, and it may not be necessary to show in vitro stability if in vivo data are available. However, due to the significantly higher effort and cost to obtain clinical data, it is efficient to optimize sensor stability in vitro and, once the desired level of stability is achieved, only then to advance to the clinical stage.

Clinical data for 14-day stability have been shown previously using a sensor based on Wired Enzyme chemistry ${ }^{10}$ leading to the development of FreeStyle Libre. More recently, a clinical trial has been conducted using actual FreeStyle Libre sensors to evaluate accuracy of the system over a 14-day wear period. Seventy-two subjects with diabetes wore two sensors simultaneously on the back of the upper arm. Capillary BG was measured by the subjects throughout the test using the built-in FreeStyle Precision Strip Port, and compared with the glucose value reported by the factory-calibrated sensor system. The BG readings on the built-in meter are independent of, and do not influence, sensor readings. ${ }^{12}$

Figure 2 shows an analysis of the 14-day stability of the sensor signal. A sensitivity value is calculated from each sensor/reference BG paired data point. For each sensor, the median of these individual sensitivity values are used to normalize data. Per-sensor normalized sensitivity values were then calculated for each day. Figure 2 shows the daily medians, interquartile ranges, and the 5 th and 95 th percentiles. That analysis illustrates any significant trends in the sensor sensitivity over the 14 days. We see a lower value on the first day, which is presumably related to the insertion process of the sensor and the associated trauma. From day 2 throughout day 14 , the median sensor sensitivity remains 


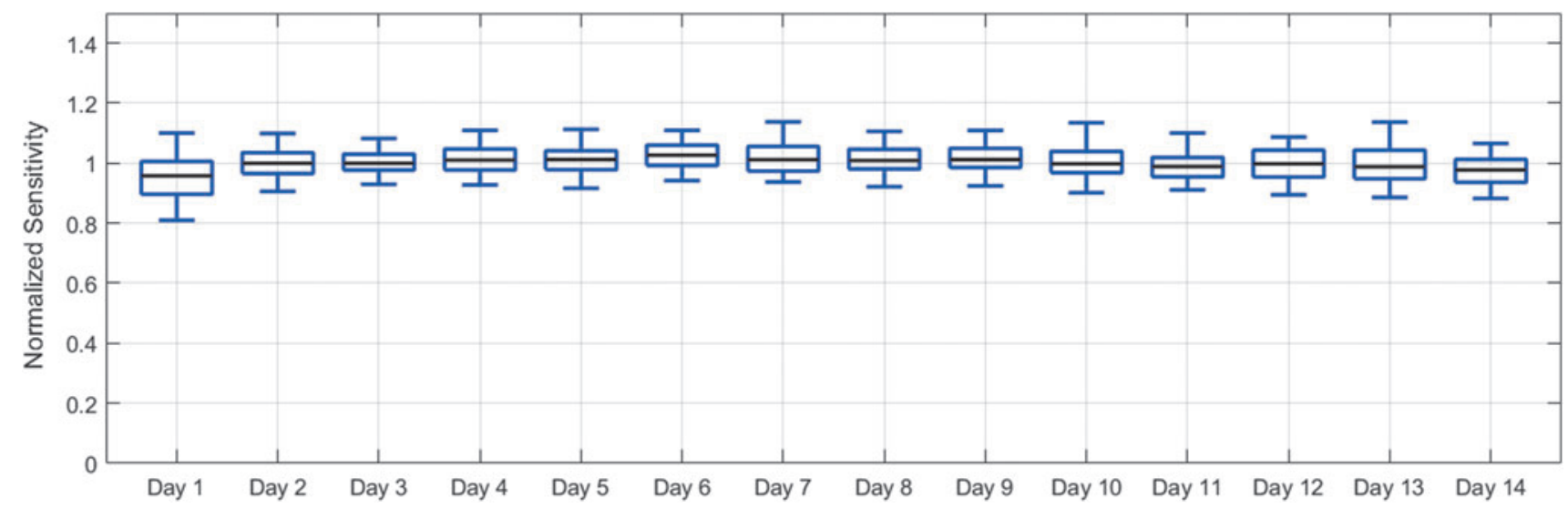

FIG. 2. Per-sensor percentile (5th, 25th, 50th, 75th, and 95th) distribution of normalized sensitivity by day. Data from 72 subjects wearing 2 sensors simultaneously were collected together with capillary BG values over a 14-day sensor wear period to calculate the in vivo sensitivities.

constant, reflecting stable sensor chemistry, as well as negligible interference from the foreign body response.

The last requirement for the feasibility of factory calibration as laid out in Table 1 is the need for a constant blood to tissue glucose relationship. This requirement can be tested by using glucose sensors with identical in vitro response to glucose in different subjects and comparing the resulting sensor sensitivities from the in vivo testing. If there was a wide distribution of the ratio of tissue to BG concentration, there would be a wide distribution of the resulting sensor sensitivities. We have previously published data supporting the hypotheses that there is no difference in the tissue to BG ratio within a person at different body sites (arm vs. abdomen) as well as between subjects. ${ }^{9,11}$ We also used data from the clinical study described earlier ${ }^{12}$ and analyzed the sensor data for their sensitivity variation. Sensors with a minimum wear duration of 10 days were included in the analysis. Figure 3 shows the results in a cumulative distribution function plot, separated by the three lots used in the study. We can see that the three lots have $80 \%-92 \%$ of their values within $10 \%$ of their respective median and $100 \%$ of the values are within $20 \%$. There are many factors that influence the calculation of each sensor's in vivo sensitivity. Errors related to BG measurements, ${ }^{40,41}$ transient sensor effects, ${ }^{31,32,42,43}$ intersensor sensitivity variation used in the study, and variations in each study subject's BG range and $B G$ rate of change range ${ }^{44}$ can contribute to the variability observed in Figure 3. The narrow distribution indicates that the tissue to BG ratio is similar between subjects, which is required for factory calibration of sensors measuring glucose in the interstitial tissue.

\section{Alternative Approaches to Sensor Calibration}

If factory calibration is not feasible, there are other options to reduce the number of BG tests required for sensor calibration. Commercially available nonfactory calibrated continuous glucose monitoring systems require a minimum of two recalibrations per day and several studies suggest that accuracy can be impacted by increasing or decreasing this frequency. ${ }^{14,20}$ As previously outlined, the frequency of recalibrations is determined by the stability of the sensor over the wear period. Increasing sensor stability can, therefore, allow for a reduction in recalibration frequency for example, once a day instead of twice a day.

If sensor stability can be guaranteed throughout the sensor wear time no recalibrations may be necessary, and calibration is only needed at the beginning of sensor wear. This approach has significant risk since the calibration factor applied to the sensor throughout its wear time will be determined through only one calibration event. Some sensor systems take a hybrid approach with a robust initial calibration (multiple

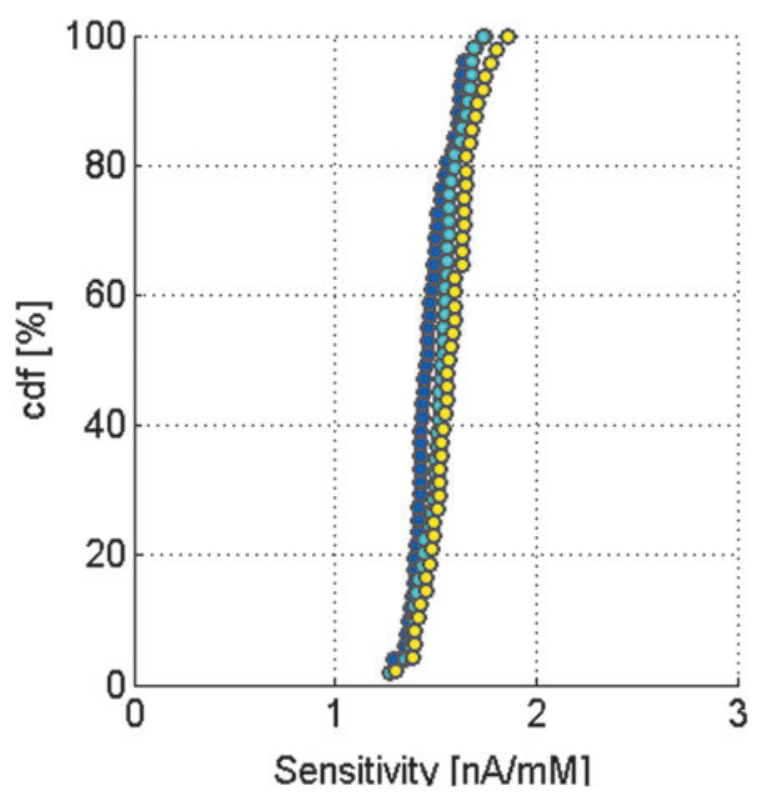

FIG. 3. Per-sensor in vivo sensitivities from three sensor lots are presented as separate distributions. Each dot represents one sensor. The in vivo sensitivity values (horizontal axis, sensor signal in $\mathrm{nA}$ divided by glucose concentration in $\mathrm{mM}$ ) for the sensors are sorted from the lowest to the highest in a cumulative distribution function (cdf).The midpoint of the sorted values on the vertical axis (50th percentile) is the median value. For each of the three lots, all of the sensor sensitivities are within $20 \%$ of their corresponding median value. 
fingerstick requests) and a reduced frequency of recalibrations (e.g., once every 2 days) ${ }^{29,45}$ to minimize the overall number of BG tests required. However, many factors can impact the reliability of fingerstick calibration, resulting in calibration being one of the more dominant sources of sensor error. $^{22,46}$ In daily use, recalibration requests may be skipped or not promptly entered, ${ }^{47,48}$ and there are many practical and technical factors ${ }^{40,41,49}$ limiting BG accuracy. ${ }^{50-53}$ A true factory calibration, where the sensor sensitivity is determined under laboratory conditions, is not susceptible to these usedependent factors.

\section{Conclusions}

The availability of factory-calibrated glucose sensors has been predicted several years ago: “... I can see the day when accuracy will be sufficient that regulators will accept that CGM values can be used for clinical decision making, that factory calibration will be possible, that reimbursement will be a foregone conclusion, and usage will be routine so that all patients and providers will need to know how to accomplish it" $\left(\right.$ Skyler $\left.^{2}\right)$. With the introduction of the FreeStyle Libre flash continuous glucose monitoring system, part of this vision has become a reality. There is no need for the user to perform BG tests for sensor calibration. Calibrations performed by the user are not only a hassle and painful, but they introduce additional cost and can also lead to inaccurate sensor readings if done incorrectly. Factory calibration is performed under laboratory conditions and is part of the sensor manufacturing process. However, to be able to implement factory calibration several requirements related to sensor stability and reproducibility have to be demonstrated and maintained over the product life.

\section{Author Disclosures Statement}

U.H. and E.B. are employees of Abbott Diabetes Care.

\section{References}

1. Garg SK: Role of emerging new technologies. Diabetes Technol Ther 2008;10:413-414.

2. Skyler JS: CGM-a technology in evolution. Diabetes Technol Ther 2009;11:63-64.

3. Mastrototaro JJ: The MiniMed continuous glucose monitoring system. Diabetes Technol Ther 2000;2 Suppl 1:S13-S18.

4. Reach G, Wilson GS: Can continuous glucose monitoring be used for the treatment of diabetes. Anal Chem 1992;64: 381A-386A.

5. Gough DA, Armour JC, Baker DA: Advances and prospects in glucose assay technology. Diabetologia 1997;40: S102-S107.

6. Wisniewski N, Moussy F, Reichert WM: Characterization of implantable biosensor membrane biofouling. Fresenius J Anal Chem 2000;366:611-621.

7. Rebrin K, Steil GM, van Antwerp WP, Mastrototaro JJ. Subcutaneous glucose predicts plasma glucose independent of insulin: implications for continuous monitoring. Am J Physiol Endocrinol Metab 1999;277:E561-E571.

8. Thomas K, Kiwit M, Kerner W: Glucose concentration in human subcutaneous adipose tissue: comparison between forearm and abdomen. Exp Clin Endocrinol Diabetes 1998; 106:465-469.
9. Hoss U, Jeddi I, Schulz M, et al.: Continuous glucose monitoring in subcutaneous tissue using factory-calibrated sensors: a pilot study. Diabetes Technol Ther 2010;12:591-597.

10. Hoss U, Budiman ES, Liu H, Christiansen MP: Continuous glucose monitoring in the subcutaneous tissue over a 14day sensor wear period. J Diabetes Sci Technol 2013;7: 1210-1219.

11. Hoss U, Budiman ES, Liu H, Christiansen MP: Feasibility of factory calibration for subcutaneous glucose sensors in subjects with diabetes. J Diabetes Sci Technol 2014;8: 89-94.

12. Bailey T, Bode BW, Christiansen MP, et al.: The performance and usability of a factory-calibrated flash glucose monitoring system. Diabetes Technol Ther 2015;17:787794.

13. Ji L, Guo X, Guo L, et al.: A multicenter evaluation of the performance and usability of a novel glucose monitoring system in Chinese adults with diabetes. J Diabetes Sci Technol 2017;11:290-295.

14. Choleau C, Klein JC, Reach G, et al.: Calibration of a subcutaneous amperometric glucose sensor implanted for 7 days in diabetic patients: Part 2. Superiority of the onepoint calibration method. Biosens Bioelectron 2002;17: 647-654.

15. Rossetti P, Bondia J, Vehí J, Fanelli CG: Estimating plasma glucose from interstitial glucose: the issue of calibration algorithms in commercial continuous glucose monitoring devices. Sensors 2010;10:10936-10952.

16. Keenan DB, Cartaya R, Mastrototaro JJ: Accuracy of a new real-time continuous glucose monitoring algorithm. J Diabetes Sci Technol 2010;4:111-118.

17. Mahmoudi Z, Johansen MD, Christiansen JS, Hejlesen O: Comparison between one-point calibration and two-point calibration approaches in a continuous glucose monitoring algorithm. J Diabetes Sci Technol 2014;8:709-719.

18. Bailey TS, Ahmann A, Brazg R, et al.: Accuracy and acceptability of the 6-day Enlite continuous subcutaneous glucose sensor. Diabetes Technol Ther 2014;16:277-283.

19. Poitout V, Moatti-Sirat D, Reach G: Calibration in dogs of a subcutaneous miniaturized glucose sensor using a glucose meter for blood glucose determination. Biosens Bioelectron 1992;7:587-592.

20. DirecNet. Evaluation of factors affecting CGMS calibration. Diabetes Technol Ther 2006;8:318-325.

21. Facchinetti A, Sparacino G, Cobelli C: Reconstruction of glucose in plasma from interstitial fluid continuous glucose monitoring data: role of sensor calibration. J Diabetes Sci Technol 2007;1:617-623.

22. Kamath A, Mahalingam A, Brauker J: Analysis of time lags and other sources of error of the DexCom SEVEN continuous glucose monitor. Diabetes Technol Ther 2009;11: 689-695.

23. Bequette BW: Continuous glucose monitoring: real-time algorithms for calibration, filtering, and alarms. J Diabetes Sci Technol 2010;4:404-418.

24. Iscoe KE, Davey RJ, Fournier PA: Do different glucose levels at calibration influence accuracy of continuous glucose monitoring readings in vitro? J Diabetes Sci Technol 2012;6:477-478.

25. Welsh JB, Kaufman FR, Lee SW: Accuracy of the sofsensor glucose sensor with the iPro calibration algorithm. J Diabetes Sci Technol 2012;6:475-476.

26. Garcia A, Rack-Gomer AL, Bhavaraju NC, et al.: Dexcom G4AP: An advanced continuous glucose monitor for the 
artificial pancreas. J Diabetes Sci Technol 2013;7:14361445.

27. Leelarathna L, English SW, Thabit H, et al.: Accuracy of subcutaneous continuous glucose monitoring in critically ill adults: improved sensor performance with enhanced calibrations. Diabetes Technol Ther 2014;16:97-101.

28. Acciaroli G, Vettoretti M, Facchinetti A, et al.: From two to one per day calibration of Dexcom G4 platinum by a timevarying day-specific bayesian prior. Diabetes Technol Ther 2016;18:472-479.

29. McGarraugh G, Brazg R, Weinstein R: FreeStyle navigator continuous glucose monitoring system with TRUstart algorithm, a 1-hour warm-up time. J Diabetes Sci Technol 2011;5:99-106.

30. King C, Anderson SM, Breton M, et al.: Modeling of calibration effectiveness and blood-to-interstitial glucose dynamics as potential confounders of the accuracy of continuous glucose sensors during hyperinsulinemic clamp. J Diabetes Sci Technol 2007;1:317-325.

31. Baysal N, Cameron F, Buckingham BA, et al.: A novel method to detect Pressure-Induced Sensor Attenuations (PISA) in an artificial pancreas. J Diabetes Sci Technol 2014;8:1091-1096.

32. Emami A, Rabasa-Lhoret R, Haidar A: Enhancing glucose sensor models: modeling the drop-outs. Diabetes Technol Ther 2015; 17:420-426.

33. Schmelzeisen-Redeker G, Schoemaker M, Kirchsteiger H, et al.: Time delay of CGM sensors: relevance, causes, and countermeasures. J Diabetes Sci Technol 2015;9:10061015.

34. Bailey T, Zisser H, Chang A: New features and performance of a next-generation SEVEN-Day continuous glucose monitoring system with short lag time. Diabetes Technol Ther 2009;11:749-756.

35. Knobbe EJ, Buckingham B: The extended kalman filter for continuous glucose monitoring. Diabetes Technol Ther 2005;7:15-27.

36. Wang J: Electrochemical glucose biosensors. Chem Rev 2008;108:814-825.

37. Ward WK: How to design a biosensor. J Diabetes Sci Technol 2007;1:201-204.

38. McGarraugh G: The chemistry of commercial continuous glucose monitors. Diabetes Technol Ther 2009;11 Suppl 1:S17-S24

39. Feldman B, Brazg R, Schwartz S, Weinstein RL: A continuous glucose sensor based on wired Enzyme ${ }^{\mathrm{TM}}$ technology-results from a 3-day trial in patients with type 1 diabetes. Diabetes Technol Ther 2003;5:769-782.

40. Schmid C, Haug C, Heinemann L, Freckmann G: System accuracy of blood glucose monitoring systems: impact of use by patients and ambient conditions. Diabetes Technol Ther 2013;15:889-896.

41. Ginsberg BH: Factors affecting blood glucose monitoring: sources of errors in measurement. J Diabetes Sci Technol 2009;3:903-913.
42. Facchinetti A, Del Favero S, Sparacino G, Cobelli C: Modeling transient disconnections and compression artifacts of continuous glucose sensors. Diabetes Technol Ther 2016;18:264-272.

43. Mensh BD, Wisniewski NA, Neil BM, Burnett DR: Susceptibility of interstitial continuous glucose monitor performance to sleeping position. J Diabetes Sci Technol 2013;7:863-870.

44. Rebrin K, Jr. NFS, Steil GM: Use of subcutaneous interstitial fluid glucose to estimate blood glucose: revisiting delay and sensor offset. J Diabetes Sci Technol 2010;4: 1087-1098.

45. Weinstein RL, Schwartz SL, Brazg RL, et al.: Accuracy of the 5-day FreeStyle Navigator ${ }^{\circledR}$ continuous glucose monitoring system: comparison with frequent laboratory reference measurements. Diabetes Care 2007;30:1125-1130.

46. Facchinetti A, Del Favero S, Sparacino G, Cobelli C: Model of glucose sensor error components: identification and assessment for new Dexcom G4 generation devices. Med Biol Eng Comput 2015;53:1259-1269.

47. Lewis KR, McCrone S, Deiriggi P, Bendre S. Effectiveness of continuous glucose monitoring in children, adolescents, and young adults with poorly controlled type 1 diabetes. J Spec Pediatr Nurs 2017;22:e12162-n/a.

48. Chase HP, Kim LM, Owen SL, et al.: Continuous subcutaneous glucose monitoring in children with type 1 diabetes. Pediatrics 2001;107:222-226.

49. Blackwell M, Wheeler BJ: Clinical review: the misreporting of logbook, download, and verbal self-measured blood glucose in adults and children with type I diabetes. Acta Diabetol 2017;54:1-8.

50. Boettcher C, Dost A, Wudy SA, et al.: Accuracy of blood glucose meters for self-monitoring affects glucose control and hypoglycemia rate in children and adolescents with type 1 diabetes. Diabetes Technol Ther 2015;17:275-282.

51. Tack C, Pohlmeier H, Behnke T, et al.: Accuracy evaluation of five blood glucose monitoring systems obtained from the pharmacy: a European multicenter study with 453 subjects. Diabetes Technol Ther 2012;14:330-337.

52. Freckmann G, Schmid C, Baumstark A, et al.: System accuracy evaluation of 43 blood glucose monitoring systems for self-monitoring of blood glucose according to DIN EN ISO 15197. J Diabetes Sci Technol 2012;6:1060-1075.

53. Pleus S, Freckmann G, Rittmeyer D, et al.: Which mean absolute relative difference is acceptable with respect to ISO 15197 system accuracy limits? Stockholm, Sweden; EASD 2015, 2015.

Address correspondence to: Udo Hoss, PhD Abbott Diabetes Care 1360 South Loop Road Alameda, CA 94502

E-mail: udo.hoss@abbott.com 